\title{
SYMMETRIC EXTENSION FOR TWO-CHANNEL QUINCUNX FILTER BANKS
}

\author{
Yi Chen, Michael D. Adams, and Wu-Sheng Lu \\ Dept. of Elec. and Comp. Eng., University of Victoria, Victoria, BC, CANADA
}

\begin{abstract}
In the case of one-dimensional filter banks, symmetric extension is a commonly used technique for constructing nonexpansive transforms of finite-length sequences. In this paper, we show how symmetric extension can be extended to the case of two-dimensional filter banks based on quincunx sampling. In particular, we show how, for filter banks of this type, one can construct nonexpansive transforms for input sequences defined on arbitrary rectangular regions.
\end{abstract}

\section{INTRODUCTION}

The two-dimensional (2-D) two-channel filter bank shown in Fig. 1 can be used to compute a class of transforms that has proven extremely useful in many image processing applications. Often, such a filter bank is defined so as to operate on sequences of infinite extent. In practice, however, we almost invariably deal with sequences of finite extent. Therefore, we usually require some means for adapting filter banks to such sequences. This leads to the well known boundary filtering problem that can arise any time a finiteextent sequence is filtered. Furthermore, in many applications, it is desirable to employ a transform that is nonexpansive (i.e., maps a sequence of $N$ samples to a new sequence of no more than $N$ samples). Consequently, we seek a solution to the boundary problem that yields nonexpansive transforms.

In the case of 1-D filter banks, symmetric extension $[1,2]$ is a commonly used technique for constructing nonexpansive transforms of finite-extent sequences. In this paper, we explain how symmetric extension can be extended to the case of 2-D filter banks based on quincunx sampling. In particular, we show how, for filter banks of this type, one can construct nonexpansive transforms for input sequences defined on arbitrary rectangular regions.

The remainder of this paper is structured as follows. Section 2 briefly comments on some of the notational conventions used herein. Then, Section 3 discusses symmetry in the 2-D case, and derives a number of results related to filter banks and symmetry. These results are then used in Section 4 in order to produce our new symmetric extension algorithm. Finally, Section 5 summarizes our work and makes some closing remarks.

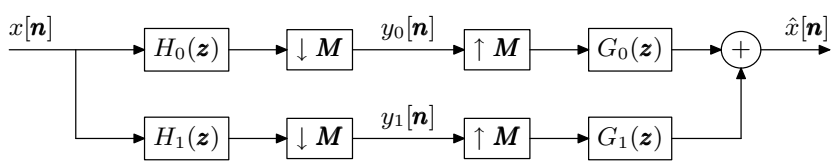

Fig. 1. 2-D two-channel filter bank.

This work was supported, in part, by the Natural Sciences and Engineering Research Council of Canada.

\section{NOTATION AND TERMINOLOGY}

Before proceeding further, a few comments are in order concerning the notation used herein. In this paper, matrices and vectors are denoted by upper and lower case boldface letters, respectively. The set of integers is denoted as $\mathbb{Z}$ (and the set of ordered pairs of integers as $\mathbb{Z}^{2}$ ). The symbol $\mathbf{1}$ is used to denote a vector/matrix of all ones, the dimensions of which should be clear from the context. The difference of two sets $A$ and $B$ is denoted $A \backslash B$. The convolution of two sequences $x$ and $y$ is denoted as $x * y$. For the most part, the multidimensional multirate systems notation employed in this paper follows that used in [3]. An element of the sequence $x$ defined on $\mathbb{Z}^{2}$ is denoted either as $x[\boldsymbol{n}]$ or $x\left[n_{0}, n_{1}\right]$ (whichever is more convenient), where $\boldsymbol{n}=\left[\begin{array}{ll}n_{0} & n_{1}\end{array}\right]^{T}$ and $n_{0}, n_{1} \in \mathbb{Z}$. The Schur product (i.e., element-wise product) of two vectors/matrices $\boldsymbol{u}$ and $\boldsymbol{v}$ is denoted $\boldsymbol{u} \circ \boldsymbol{v}$. For two vectors, $\boldsymbol{u}$ and $\boldsymbol{v}$, we write $\boldsymbol{u} \geq \boldsymbol{v}$ if every element in $\boldsymbol{u}$ is greater than or equal to its corresponding element in $\boldsymbol{v}$. The lattice generated by sampling matrix $\boldsymbol{M}$ is denoted $\operatorname{LAT}(\boldsymbol{M})$ (i.e., $\operatorname{LAT}(\boldsymbol{M})=\{\boldsymbol{M} \boldsymbol{n}\}_{\boldsymbol{n} \in \mathbb{Z}^{2}}$ ). The quincunx lattice can be associated with the generating matrix $\boldsymbol{M}=\left[\begin{array}{cc}1 & 1 \\ 1 & -1\end{array}\right]$ and the two representative coset vectors $\boldsymbol{k}_{0}=\left[\begin{array}{ll}0 & 0\end{array}\right]^{T}$ and $\boldsymbol{k}_{1}=\left[\begin{array}{ll}1 & 0\end{array}\right]^{T}$. Throughout this work, the quantity $\boldsymbol{M}$ should be understood to be this particular generating matrix of the quincunx lattice (unless explicitly noted otherwise).

\section{SYMMETRIC EXTENSION PRELIMINARIES}

With our proposed symmetric extension scheme, we use a structure for the forward transform like that shown in Fig. 2. We convert the input sequence $\tilde{x}$ to the filter bank into an infinite-extent periodic symmetric sequence $x$. This eliminates the boundary filtering problem (as $x$ is an infinite-extent sequence). Then, by carefully constraining the choice of filters $\left(H_{0}\right.$ and $\left.H_{1}\right)$, we can force the subband sequences $y_{0}$ and $y_{1}$ to always be both symmetric and periodic. Lastly, we use these symmetry and periodicity properties in order to extract only those samples from the subband sequences that are independent. With some care, it is possible for the resulting transform to be nonexpansive.

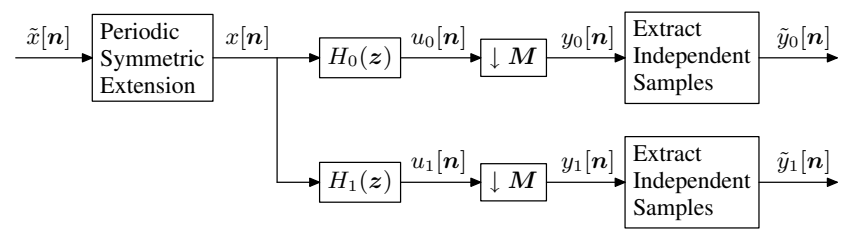

Fig. 2. Analysis side of the filter bank with symmetric extension.

The notion of symmetry (of a sequence) is of fundamental importance herein. In the 1-D case, only a very limited number of symmetry types is possible, but in the 2-D case, considerably more possibilities exist. We begin by introducing several types of symmetries relevant to this work, the first of which is given by the definition below. 
Definition 1 (Centrosymmetry). A sequence $x$ defined on $\mathbb{Z}^{2}$ is said to be centrosymmetric about $\boldsymbol{c}$ (i.e., has linear phase with group delay $\boldsymbol{c}$ ) if, for some $\boldsymbol{c} \in \frac{1}{2} \mathbb{Z}^{2}$ and $S \in\{-1,1\}$,

$$
x[\boldsymbol{n}]=S x[2 \boldsymbol{c}-\boldsymbol{n}] \quad \text { for all } \boldsymbol{n} \in \mathbb{Z}^{2} .
$$

From the preceding definition, it follows that only (approximately) half of the samples of a centrosymmetric sequence are independent. The next symmetry type of interest is given by the definition below.

Definition 2 (Quadrantal centrosymmetry). A sequence $x$ defined on $\mathbb{Z}^{2}$ is said to be quadrantally centrosymmetric about $\boldsymbol{c}$ if, for some $c \in \frac{1}{2} \mathbb{Z}^{2}$ and $A=1,2,3$,

$$
x[\boldsymbol{n}]=s[A] x[\boldsymbol{c} \circ(\mathbf{1}-\boldsymbol{v}[A])+\boldsymbol{n} \circ \boldsymbol{v}[A]] \text { for all } \boldsymbol{n} \in \mathbb{Z}^{2},
$$

where $s[A] \in\{-1,1\}, \boldsymbol{v}[A]=\left[(-1)^{a_{0}}(-1)^{a_{1}}\right]^{T}, a_{0}, a_{1} \in\{0,1\}$, and $A=a_{0}+2 a_{1}$. In terms of $s[\cdot]$ in (2), only four types of quadrantal centrosymmetry are possible [4]:

\begin{tabular}{|l|rrr|}
\hline Type & $s[1]$ & $s[2]$ & $s[3]$ \\
\hline even-even & 1 & 1 & 1 \\
odd-odd & -1 & -1 & 1 \\
even-odd & 1 & -1 & -1 \\
odd-even & -1 & 1 & -1 \\
\hline
\end{tabular}

An example of an even-even quadrantally centrosymmetric sequence with its symmetry center on the integer lattice is shown in Fig. 3(a). Clearly, quadrantal centrosymmetry is a type of fourfold symmetry, where only (approximately) $\frac{1}{4}$ of the samples are independent (e.g., those with indices $\boldsymbol{n} \geq \boldsymbol{c}$ ). In the context of this paper, the even-even type of symmetry is of most interest. Now, we define one further type of symmetry which we will need herein.

Definition 3 (Rotated quadrantal centrosymmetry). A sequence $x$ defined on $\mathbb{Z}^{2}$ is said to be rotated quadrantally centrosymmetric about $\boldsymbol{c}$ if, for some $S, T \in\{-1,1\}$ and $\boldsymbol{c}=\left[\begin{array}{ll}c_{0} & c_{1}\end{array}\right]^{T} \in \frac{1}{2} \mathbb{Z}^{2}$ satisfying $c_{0}+c_{1} \in \mathbb{Z}$,

$$
\begin{aligned}
x\left[n_{0}, n_{1}\right] & =\operatorname{Sx}\left[2 c_{0}-n_{0}, 2 c_{1}-n_{1}\right] \\
& =\operatorname{Tx}\left[c_{0}-c_{1}+n_{1}, c_{1}-c_{0}+n_{0}\right] \\
& =\operatorname{STx}\left[c_{0}+c_{1}-n_{1}, c_{0}+c_{1}-n_{0}\right]
\end{aligned}
$$

for all $n_{0}, n_{1} \in \mathbb{Z}$.

Rotated quadrantal centrosymmetry is a type of four-fold symmetry, where only approximately $\frac{1}{4}$ of the samples are independent (e.g., those with indices $\boldsymbol{n}$ satisfying $\boldsymbol{M n} \geq \boldsymbol{M c}$ ). An example of a rotated quadrantally centrosymmetric sequence with its symmetry center on the integer lattice is shown in Fig. 3(b).

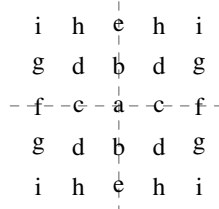

(a)

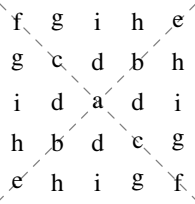

(b)
Fig. 3. Types of 4-fold symmetry. (a) Quadrantal centrosymmetry and (b) rotated quadrantal centrosymmetry.

We now introduce a scheme for mapping a finite-extent (2D) sequence defined on a rectangular region to an infinite-extent sequence that is both quadrantally centrosymmetric and periodic. This process called symmetric extension is defined as given below.
Definition 4 (Symmetric extension of sequence). Let $\tilde{x}$ be a (2D) sequence defined on the rectangular region $\left\{0,1, \ldots, L_{0}-1\right\} \times$ $\left\{0,1, \ldots, L_{1}-1\right\}$. Then, the symmetric extension $x$ of $\tilde{x}$ is defined as

where $f_{0}\left[n_{0}\right]$ and $f_{1}\left[n_{1}\right]$ are given by

$$
x\left[n_{0}, n_{1}\right]=\tilde{x}\left[f_{0}\left[n_{0}\right], f_{1}\left[n_{1}\right]\right],
$$

$$
\begin{aligned}
& f_{0}\left[n_{0}\right]=\min \left\{\bmod \left(n_{0}, 2 L_{0}-2\right), 2 L_{0}-2-\bmod \left(n_{0}, 2 L_{0}-2\right)\right\}, \\
& f_{1}\left[n_{1}\right]=\min \left\{\bmod \left(n_{1}, 2 L_{1}-2\right), 2 L_{1}-2-\bmod \left(n_{1}, 2 L_{1}-2\right)\right\} .
\end{aligned}
$$

The symmetric extension of a 2-D sequence (as defined above) can be viewed as 1-D extension operation applied along each of the dimensions of the sequence separately. For this reason, the rows (i.e., 1-D horizontal slices of the 2-D sequence) are symmetric about the vertical axis and $\left(2 L_{0}-2\right)$-periodic in the horizontal direction, and the columns (i.e., 1-D vertical slices of the 2-D sequence) are symmetric about the horizontal axis and $\left(2 L_{1}-2\right)$ periodic in the vertical direction. This leads to the 2-D symmetrically extended sequence also having symmetry and periodicity properties as elucidated by the below lemma.

Lemma 1 (Properties of symmetrically extended sequences). Let $\tilde{x}$ be a sequence defined on the rectangular region $\left\{0,1, \ldots, L_{0}-\right.$ $1\} \times\left\{0,1, \ldots, L_{1}-1\right\}$. Let $x$ denote the symmetric extension of $\tilde{x}$ as defined by (4). Then, $x$ is $\boldsymbol{P}$-periodic with $\boldsymbol{M}^{-1} \boldsymbol{P}$ being an integer matrix, and is even-even quadrantally centrosymmetric about $\mathbf{0}$. (Recall that $\boldsymbol{M}$ is as defined in Section 2.)

Proof. First, we show that $x$ is $\boldsymbol{P}$-periodic with $\boldsymbol{P}=$ $\boldsymbol{M}\left[\begin{array}{cc}L_{0}-1 & L_{1}-1 \\ L_{0}-1 & -L_{1}+1\end{array}\right]$. Since $\bmod (u+k v, v)=\bmod (u, v)$ for $k \in \mathbb{Z}$, we have $f_{0}\left[n_{0}+\left(2 L_{0}-2\right) k_{0}\right]=f_{0}\left[n_{0}\right]$ and $f_{1}\left[n_{1}+\left(2 L_{1}-2\right) k_{1}\right]=$ $f_{1}\left[n_{1}\right]$, for $k_{0}, k_{1} \in \mathbb{Z}$. This implies that $x[\boldsymbol{n}+\boldsymbol{P k}]=x[\boldsymbol{n}]$ for $\boldsymbol{k}=$ $\left[\begin{array}{ll}k_{0} & k_{1}\end{array}\right]^{T}$ with $\boldsymbol{P}=\left[\begin{array}{cc}2 L_{0}-2 & 0 \\ 0 & 2 L_{1}-2\end{array}\right]$. Therefore, $x$ is $\boldsymbol{P}$-periodic, and $\boldsymbol{M}^{-1} \boldsymbol{P}=\left[\begin{array}{cc}L_{0}-1 & L_{1}-1 \\ L_{0}-1 & -L_{1}+1\end{array}\right]$ is an integer matrix.

Now, we show that $x$ is quadrantally centrosymmetric about $\mathbf{0}$. For $u, v \in \mathbb{Z}$ and $v \nmid u, \bmod (-u, v)=v-\bmod (u, v)$. It follows that $f_{0}\left[-n_{0}\right]=f_{0}\left[n_{0}\right]$ and $f_{1}\left[-n_{1}\right]=f_{1}\left[n_{1}\right]$. Therefore,

$$
\begin{aligned}
x[\boldsymbol{n} \circ \boldsymbol{v}[A]] & =x\left[(-1)^{a_{0}} n_{0},(-1)^{a_{1}} n_{1}\right] \\
& =\tilde{x}\left[f_{0}\left[(-1)^{a_{0}} n_{0}\right], f_{1}\left[(-1)^{a_{1}} n_{1}\right]\right] \\
& =\tilde{x}\left[f_{0}\left[n_{0}\right], f_{1}\left[n_{1}\right]\right] \\
& =x[\boldsymbol{n}]
\end{aligned}
$$

for $A=1,2,3$. Thus, from Definition $2, x$ is quadrantally centrosymmetric about $\mathbf{0}$. Since $s[A]$ in (2) is 1 for $A=1,2,3, x$ has the even-even symmetry. (Due to $\boldsymbol{P}$-periodicity, $x$ is also quadrantally centrosymmetric about $\boldsymbol{P} \boldsymbol{k}$ for $\boldsymbol{k} \in \frac{1}{2} \mathbb{Z}^{2}$.)

As shown in Fig. 2, the original input sequence $\tilde{x}$ is symmetrically extended and then fed into the analysis side of the filter bank, which consists of filters followed by downsamplers. The ability to construct a nonexpansive transform then depends on the preservation of the four-fold symmetry and periodicity in the subband sequences $y_{0}$ and $y_{1}$. In what follows, we will examine how the operations of convolution and downsampling affect these properties. First, we consider the effects of convolution on symmetry as given by the below lemma.

Lemma 2 (Preservation of symmetry under convolution). Let $x$ and $h$ be sequences defined on $\mathbb{Z}^{2}$, and define $y=x * h$. If $x$ and $h$ are centrosymmetric about $\boldsymbol{c}_{x}$ and $\boldsymbol{c}_{h}$, respectively, then $y$ is centrosymmetric about $\boldsymbol{c}_{y}=\boldsymbol{c}_{x}+\boldsymbol{c}_{h}$. If $x$ and $h$ are quadrantally centrosymmetric about $\boldsymbol{c}_{x}$ and $\boldsymbol{c}_{h}$, respectively, then $y$ is quadrantally centrosymmetric about $\boldsymbol{c}_{y}=\boldsymbol{c}_{x}+\boldsymbol{c}_{h}$. 
Proof. The proof for the centrosymmetric case is essentially the same as in the 1-D case, so we omit it here in the interest of saving space. Now, we consider the quadrantally centrosymmetric case. We have

$$
y[\boldsymbol{n}]=\sum_{k \in \mathbb{Z}^{2}} x[\boldsymbol{k}] h[\boldsymbol{n}-\boldsymbol{k}] .
$$

Since $x$ and $h$ are quadrantally centrosymmetric about $\boldsymbol{c}_{x}$ and $\boldsymbol{c}_{h}$, respectively, we have

$$
\begin{aligned}
& x[\boldsymbol{n}]=s_{x}[A] x\left[\boldsymbol{c}_{x} \circ(1-\boldsymbol{v}[A])+\boldsymbol{n} \circ \boldsymbol{v}[A]\right] \quad \text { and } \\
& h[\boldsymbol{n}]=s_{h}[A] h\left[\boldsymbol{c}_{h} \circ(1-\boldsymbol{v}[A])+\boldsymbol{n} \circ \boldsymbol{v}[A]\right],
\end{aligned}
$$

for all $\boldsymbol{n} \in \mathbb{Z}^{2}$. Substituting (6) and (7) into (5), we obtain

$$
\begin{aligned}
y[\boldsymbol{n}]= & \sum_{k \in \mathbb{Z}^{2}} s_{x}[A] x\left[\boldsymbol{c}_{x} \circ(1-\boldsymbol{v}[A])+\boldsymbol{k} \circ \boldsymbol{v}[A]\right] \\
& s_{h}[A] h\left[\boldsymbol{c}_{h} \circ(1-\boldsymbol{v}[A])+(\boldsymbol{n}-\boldsymbol{k}) \circ \boldsymbol{v}[A]\right] .
\end{aligned}
$$

Let $\boldsymbol{k}^{\prime}=\boldsymbol{c}_{x} \circ(1-\boldsymbol{v}[A])+\boldsymbol{k} \circ \boldsymbol{v}[A]$. Solving for $\boldsymbol{k}$ in terms of $\boldsymbol{k}^{\prime}$ yields $\boldsymbol{k}=\boldsymbol{c}_{x} \circ(1-\boldsymbol{v}[A])+\boldsymbol{k}^{\prime} \circ \boldsymbol{v}[A]$. Applying the change of variable to (8), we obtain (for $A=1,2,3$ )

$$
\begin{aligned}
y[\boldsymbol{n}]= & \sum_{\boldsymbol{k}^{\prime} \in \mathbb{Z}^{2}} s_{x}[A] x\left[\boldsymbol{k}^{\prime}\right] s_{h}[A] h\left[\boldsymbol{c}_{h} \circ(1-\boldsymbol{v}[A])\right. \\
& \left.\quad+\left(\boldsymbol{n}-\left\{\boldsymbol{c}_{x} \circ(1-\boldsymbol{v}[A])+\boldsymbol{k}^{\prime} \circ \boldsymbol{v}[A]\right\}\right) \circ \boldsymbol{v}[A]\right] \\
= & \sum_{\boldsymbol{k}^{\prime} \in \mathbb{Z}^{2}} s_{x}[A] x\left[\boldsymbol{k}^{\prime}\right] s_{h}[A] h\left[\boldsymbol{c}_{h} \circ(1-\boldsymbol{v}[A])+\boldsymbol{n} \circ \boldsymbol{v}[A]\right. \\
& \left.\quad-\boldsymbol{k}^{\prime}-\boldsymbol{c}_{x} \circ(1-\boldsymbol{v}[A]) \circ \boldsymbol{v}[A]\right] \\
= & s_{x}[A] s_{h}[A] \sum_{\boldsymbol{k} \in \mathbb{Z}^{2}} x[\boldsymbol{k}] h\left[\left(\boldsymbol{c}_{h}+\boldsymbol{c}_{x}\right) \circ(1-\boldsymbol{v}[A])\right. \\
& \quad+\boldsymbol{n} \circ \boldsymbol{v}[A]-\boldsymbol{k}] \\
= & s_{y}[A] \sum_{\boldsymbol{k} \in \mathbb{Z}^{2}} x[\boldsymbol{k}] h\left[\left(\boldsymbol{c}_{x}+\boldsymbol{c}_{h}\right) \circ(1-\boldsymbol{v}[A])+\boldsymbol{n} \circ \boldsymbol{v}[A]-\boldsymbol{k}\right] \\
= & s_{y}[A] y\left[\left(\boldsymbol{c}_{x}+\boldsymbol{c}_{h}\right) \circ(1-\boldsymbol{v}[A])+\boldsymbol{n} \circ \boldsymbol{v}[A]\right],
\end{aligned}
$$

where $s_{y}[A]=s_{x}[A] s_{h}[A]$. As $s_{x}[\cdot]$ and $s_{h}[\cdot]$ each corresponds to one row of the table in Definition $2, s_{y}[\cdot]$ is also in that table. Thus, $y$ is quadrantally centrosymmetric about $\boldsymbol{c}_{y}=\boldsymbol{c}_{x}+\boldsymbol{c}_{h}$.

Now, we consider the effect of convolution on periodicity in the lemma below.

Lemma 3 (Preservation of periodicity under convolution). Let $x$ and $h$ be sequences defined on $\mathbb{Z}^{2}$, with $x$ being $\boldsymbol{P}$-periodic. Then, $y=x * h$ is $\boldsymbol{P}$-periodic [5].

Next, we examine the effects of downsampling on periodicity and symmetry. First, we consider the case of periodicity in the lemma below.

Lemma 4 (Downsampling of periodic sequence). Let $\boldsymbol{M}$ be an arbitrary sampling matrix. Let $x$ be a $\boldsymbol{P}$-periodic sequence such that $\boldsymbol{M}^{-1} \boldsymbol{P}$ is an integer matrix. Then, $(\downarrow \boldsymbol{M}) x$ is $\left(\boldsymbol{M}^{-1} \boldsymbol{P}\right)$-periodic.

Proof. Since $x$ is $\boldsymbol{P}$-periodic, $x[\boldsymbol{n}]=x[\boldsymbol{n}+\boldsymbol{P} \boldsymbol{k}]$ for $\boldsymbol{k} \in \mathbb{Z}^{2}$. The downsampled sequence $y$ is given by

$$
\begin{aligned}
y[\boldsymbol{n}] & =(\downarrow \boldsymbol{M}) x[\boldsymbol{n}] \\
& =x[\boldsymbol{M n}] \\
& =x[\boldsymbol{M n}+\boldsymbol{P k}] \\
& =x\left[\boldsymbol{M}\left(\boldsymbol{n}+\left(\boldsymbol{M}^{-1} \boldsymbol{P}\right) \boldsymbol{k}\right)\right] .
\end{aligned}
$$

Since $\boldsymbol{M}^{-1} \boldsymbol{P}$ is an integer matrix, we have

$$
x\left[\boldsymbol{M}\left(\boldsymbol{n}+\left(\boldsymbol{M}^{-1} \boldsymbol{P}\right) \boldsymbol{k}\right)\right]=y\left[\boldsymbol{n}+\left(\boldsymbol{M}^{-1} \boldsymbol{P}\right) \boldsymbol{k}\right] .
$$

Substituting (10) into (9), we have that $y[\boldsymbol{n}]=y\left[\boldsymbol{n}+\left(\boldsymbol{M}^{-1} \boldsymbol{P}\right) \boldsymbol{k}\right]$ for $\boldsymbol{k} \in \mathbb{Z}^{2}$. Therefore, $y$ is $\boldsymbol{M}^{-1} \boldsymbol{P}$-periodic.
Thus, from above, if a $\boldsymbol{P}$-periodic sequence has both of its periodicity vectors (i.e., columns of $\boldsymbol{P}$ ) on $\operatorname{LAT}(\boldsymbol{M})$, the sequence downsampled by $\boldsymbol{M}$ is periodic with the number of samples in one period being reduced by a factor of $|\operatorname{det} \boldsymbol{M}|$ relative to the original sequence. Next, we consider the effects of downsampling on symmetry, as elucidated by the lemma below.

Lemma 5 (Downsampling of quadrantally centrosymmetric sequence). Let $x$ be a quadrantally centrosymmetric sequence with symmetry center $\boldsymbol{c}_{x} \in \mathbb{Z}^{2}$. Define $y=(\downarrow \boldsymbol{M}) x$. Then, $y$ is rotated quadrantally centrosymmetric about $\boldsymbol{M}^{-1} \boldsymbol{c}_{x}$. (Recall that $\boldsymbol{M}$ is as defined in Section 2.)

Proof. The downsampled sequence $y$ is given by

$$
y\left[n_{0}, n_{1}\right]=(\downarrow \boldsymbol{M}) x[\boldsymbol{n}]=x\left[n_{0}+n_{1}, n_{0}-n_{1}\right] .
$$

As $x$ is quadrantally centrosymmetric about $\boldsymbol{c}_{x}=\left[\begin{array}{ll}c_{0} & c_{1}\end{array}\right]^{T}$, we have

$$
\begin{aligned}
& x\left[n_{0}+n_{1}, n_{0}-n_{1}\right] \\
= & S x\left[2 c_{0}-n_{0}-n_{1}, 2 c_{1}-n_{0}+n_{1}\right] \\
= & \operatorname{Tx}\left[2 c_{0}-n_{0}-n_{1}, n_{0}-n_{1}\right] \\
= & S T x\left[n_{0}+n_{1}, 2 c_{1}-n_{0}+n_{1}\right]
\end{aligned}
$$

for $S, T \in\{-1,1\}$. Since $c_{0}, c_{1} \in \mathbb{Z}$, we have

$$
\begin{aligned}
x\left[2 c_{0}-n_{0}-n_{1}, 2 c_{1}-n_{0}+n_{1}\right] & =y\left[c_{0}+c_{1}-n_{0}, c_{0}-c_{1}-n_{1}\right] \\
x\left[2 c_{0}-n_{0}-n_{1}, n_{0}-n_{1}\right] & =y\left[c_{0}-n_{1}, c_{0}-n_{0}\right] \\
x\left[n_{0}+n_{1}, 2 c_{1}-n_{0}+n_{1}\right] & =y\left[n_{1}+c_{1}, n_{0}-c_{1}\right] .
\end{aligned}
$$

Substituting (11) and the above three equations into (12), we obtain

$$
\begin{aligned}
y\left[n_{0}, n_{1}\right] & =\operatorname{Sy}\left[c_{0}+c_{1}-n_{0}, c_{0}-c_{1}-n_{1}\right] \\
& =\operatorname{Ty}\left[c_{0}-n_{1}, c_{0}-n_{0}\right] \\
& =\operatorname{STy}\left[n_{1}+c_{1}, n_{0}-c_{1}\right] .
\end{aligned}
$$

Therefore, the downsampled sequence $y$ has rotated quadrantal centrosymmetry about $\boldsymbol{M}^{-1} \boldsymbol{c}_{x}=\left[\begin{array}{ll}\frac{c_{0}+c_{1}}{2} & \frac{c_{0}-c_{1}}{2}\end{array}\right]^{T}$.

The preceding lemma shows that if the symmetry center $\boldsymbol{c}_{x}$ of a quadrantally centrosymmetric sequence $x$ is on the integer lattice, then the downsampled sequence $y$ also has four-fold symmetry, as each set of dependent coefficients in $x$ is contained in the same coset of the quincunx lattice. Thus, the number of independent samples in $y$ is about half of that of $x$.

\section{SYMMETRIC EXTENSION ALGORITHM}

Using our previous results, we will now derive a scheme based on symmetric extension that allows for the construction of nonexpansive transforms based on a two-channel quincunx filter bank. To begin, we recall the perfect reconstruction (PR) condition for such a filter bank is given by

$$
\begin{gathered}
H_{0}(z) G_{0}(z)+H_{1}(z) G_{1}(z)=2 z^{-l} \text { and } \\
H_{0}(-z) G_{0}(z)+H_{1}(-z) G_{1}(z)=0,
\end{gathered}
$$

where $H_{0}(z)$ and $H_{1}(z)$ are the analysis filter transfer functions, $G_{0}(z)$ and $G_{1}(z)$ are the synthesis filter transfer functions, $\boldsymbol{l}=$ $\left[\begin{array}{ll}l_{0} & l_{1}\end{array}\right]^{T} \in \mathbb{Z}^{2}$, and $z^{-\boldsymbol{l}}=z_{0}^{-l_{0}} z_{1}^{-l_{1}}$. Letting $G_{0}(z)=H_{1}(-z), G_{1}(z)=$ $-H_{0}(-\boldsymbol{z})$, and defining $P(\boldsymbol{z})=H_{0}(\boldsymbol{z}) G_{0}(\boldsymbol{z})=$ $H_{0}(\boldsymbol{z}) H_{1}(-\boldsymbol{z})$, the PR condition becomes $P(\boldsymbol{z})-P(-\boldsymbol{z})=2 \boldsymbol{z}^{-\boldsymbol{l}}$. If $H_{0}$ and $H_{1}$ have quadrantal centrosymmetry with group delays $\boldsymbol{d}_{0}$ and $\boldsymbol{d}_{1}$ respectively, then $P(\boldsymbol{z})$ and $P(-\boldsymbol{z})$ are also quadrantally centrosymmetric with group delay $\boldsymbol{d}_{p}=\boldsymbol{d}_{0}+\boldsymbol{d}_{1}$. 
In this paper, we consider the case where both analysis filters have group delays on the integer lattice. We can see that $\boldsymbol{d}_{0}$ and $\boldsymbol{d}_{1}$ must be in different cosets of the quincunx lattice, otherwise $\boldsymbol{d}_{p} \in$ LAT $(\boldsymbol{M}), P(\boldsymbol{z})-P(-\boldsymbol{z})$ cannot be a monomial. Furthermore, we can also conclude that for this case only filters with even-even type of symmetry satisfy the PR condition. These observations suggest that such a perfect reconstruction filter bank having analysis filters with group delays in $\mathbb{Z}^{2}$ is compatible with symmetric extension. This leads to the main result of our paper which is as follows:

Theorem 1 (Symmetric extension algorithm). Consider the filter bank shown in Fig. 2, where $\tilde{x}$ is defined on the rectangular region $\left\{0,1, \ldots, L_{0}-1\right\} \times\left\{0,1, \ldots, L_{1}-1\right\}$ and $x$ is the symmetric extension of $\tilde{x}$ as given by (4). If $H_{0}$ and $H_{1}$ are quadrantally centrosymmetric with group delays $\boldsymbol{d}_{0}=\left[\begin{array}{ll}d_{0,0} & d_{0,1}\end{array}\right]^{T} \in \mathbb{Z}^{2}$ and $\boldsymbol{d}_{1}=\left[\begin{array}{ll}d_{1,0} & d_{1,1}\end{array}\right]^{T} \in \mathbb{Z}^{2}$, respectively, then the subband output $y_{0}$ can be completely characterized by $N_{0}$ samples with indices $\boldsymbol{n}=\left[\begin{array}{ll}n_{0} & n_{1}\end{array}\right]^{T}$ given by

$$
\begin{array}{r}
\left\lceil\frac{d_{0,0}+d_{0,1}}{2}\right\rceil \leq n_{0} \leq\left\lfloor\frac{d_{0,0}+d_{0,1}+L_{0}+L_{1}}{2}\right\rfloor-1, \\
\text { and } \max \left\{d_{0,0}-n_{0}, n_{0}-d_{0,1}-L_{1}+1\right\} \leq n_{1} \\
\leq \min \left\{d_{0,0}+L_{0}-1-n_{0}, n_{0}-d_{0,1}\right\} ;
\end{array}
$$

$y_{1}$ can be completely characterized by $N_{1}$ samples with indices $\boldsymbol{n}=\left[\begin{array}{ll}n_{0} & n_{1}\end{array}\right]^{T}$ given by

$$
\begin{array}{r}
\left\lceil\frac{d_{1,0}+d_{1,1}}{2}\right\rceil \leq n_{0} \leq\left\lfloor\frac{d_{1,0}+d_{1,1}+L_{0}+L_{1}}{2}\right\rfloor-1, \\
\text { and } \max \left\{d_{1,0}-n_{0}, n_{0}-d_{1,1}-L_{1}+1\right\} \leq n_{1} \\
\leq \min \left\{d_{1,0}+L_{0}-1-n_{0}, n_{0}-d_{1,1}\right\} ;
\end{array}
$$

and $N_{0}+N_{1}=L_{0} L_{1}$ (i.e., the transform is nonexpansive).

Proof. In what follows, we refer to signals in Fig. 2. From Lemma 1, we know that $x$ is $\boldsymbol{P}$-periodic with $\boldsymbol{P}=\left[\begin{array}{cc}2 L_{0}-2 & 0 \\ 0 & 2 L_{1}-2\end{array}\right]$, and is quadrantally centrosymmetric about $\mathbf{0}$. Consider the first channel, where $H_{0}$ is quadrantally centrosymmetric with group delay $\boldsymbol{d}_{0} \in \mathbb{Z}^{2}$. Then, the analysis filter output $u_{0}$ is $\boldsymbol{P}$-periodic from Lemma 3 , and quadrantally centrosymmetric about $\mathbf{0}+\boldsymbol{d}_{0}=\boldsymbol{d}_{0}$ from Lemma 2 . Since $\boldsymbol{M}^{-1} \boldsymbol{P}=\left[\begin{array}{cc}L_{0}-1 & L_{1}-1 \\ L_{0}-1 & -L_{1}+1\end{array}\right]$ is an integer matrix and $\boldsymbol{d}_{0} \in \mathbb{Z}^{2}, y_{0}$ is $\boldsymbol{M}^{-1} \boldsymbol{P}$-periodic from Lemma 4 , and rotated quadrantally centrosymmetric about $\boldsymbol{M}^{-1} \boldsymbol{d}_{0}$ from Lemma 5.

Therefore, $y_{0}$ can be completely characterized by samples with indices $\boldsymbol{n}=\left[\begin{array}{ll}n_{0} & n_{1}\end{array}\right]^{T}$ given by

$$
\begin{gathered}
\boldsymbol{M n} \in\left\{d_{0,0}, d_{0,0}+1, \ldots, d_{0,0}+L_{0}-1\right\} \times \\
\left\{d_{0,1}, d_{0,1}+1, \ldots, d_{0,1}+L_{1}-1\right\} .
\end{gathered}
$$

Solving (15), we obtain the conditions for $n_{0}$ and $n_{1}$ as shown in (13). The number $N_{0}$ of characteristic samples of $y_{0}$ is given by

$$
N_{0}= \begin{cases}\frac{1}{2} L_{0} L_{1} & \text { for } L_{0} L_{1} \text { even } \\ \frac{1}{2}\left(L_{0} L_{1}+1\right) & \text { for } L_{0} L_{1} \text { odd, } \boldsymbol{d}_{0} \in \operatorname{LAT}(\boldsymbol{M}) \\ \frac{1}{2}\left(L_{0} L_{1}-1\right) & \text { for } L_{0} L_{1} \text { odd, } \boldsymbol{d}_{0} \notin \operatorname{LAT}(\boldsymbol{M})\end{cases}
$$

which can be equivalently written as

$$
N_{0}=\left\lfloor\frac{1}{2}\left(L_{0} L_{1}+d_{0,0}+d_{0,1}+1\right)\right\rfloor-\left\lceil\frac{1}{2}\left(d_{0,0}+d_{0,1}\right)\right\rceil .
$$

Similarly, $y_{1}$ is characterized by samples with indices $\boldsymbol{n}=$ $\left[\begin{array}{ll}n_{0} & n_{1}\end{array}\right]^{T}$ given by (14). The number $N_{1}$ of characteristic samples of $y_{1}$ is given by

$$
N_{1}= \begin{cases}\frac{1}{2} L_{0} L_{1} & \text { for } L_{0} L_{1} \text { even } \\ \frac{1}{2}\left(L_{0} L_{1}+1\right) & \text { for } L_{0} L_{1} \text { odd, } \boldsymbol{d}_{1} \in \operatorname{LAT}(\boldsymbol{M}) \\ \frac{1}{2}\left(L_{0} L_{1}-1\right) & \text { for } L_{0} L_{1} \text { odd, } \boldsymbol{d}_{1} \notin \operatorname{LAT}(\boldsymbol{M}) .\end{cases}
$$

Since only one of $\boldsymbol{d}_{0}$ and $\boldsymbol{d}_{1}$ is on the quincunx lattice, from (16) and (17), we have $N_{0}+N_{1}=L_{0} L_{1}$.

The significance of the above theorem is that it shows how one can construct a nonexpansive transform from a two-channel quincunx filter bank. There are also other types of PR filter banks that lead to nonexpansive transforms with slight variations on the above algorithm. In what follows, we briefly comment on two such variations. For additional details, the reader is referred to [6].

Let $H_{0}$ and $H_{1}$ be quadrantally centrosymmetric analysis filters with group delays $\boldsymbol{d}_{0}=\left[\begin{array}{ll}d_{0,0} & d_{0,1}\end{array}\right]^{T}$ and $\boldsymbol{d}_{1}=\left[\begin{array}{ll}d_{1,0} & d_{1,1}\end{array}\right]^{T}$, respectively. If $H_{0}$ has even-even symmetry, $d_{0,0} \in \frac{1}{2} \backslash \mathbb{Z} \backslash \mathbb{Z}$ and $d_{0,1} \in \mathbb{Z}, H_{1}$ has odd-even symmetry, $d_{1,0} \in \frac{1}{2} \mathbb{Z} \backslash \mathbb{Z}$ and $d_{1,1} \in \mathbb{Z}$, then this filter bank is compatible with symmetric extension, except that in (4), $f_{0}$ is instead given by

$$
f_{0}\left[n_{0}\right]=\min \left\{\bmod \left(n_{0}, 2 L_{0}\right), 2 L_{0}-1-\bmod \left(n_{0}, 2 L_{0}\right)\right\} .
$$

With this symmetric extension, $x$ is quadrantally centrosymmetric about $\left[\begin{array}{ll}-\frac{1}{2} & 0\end{array}\right]^{T}$, and is $\boldsymbol{P}$-periodic with $\boldsymbol{P}=\left[\begin{array}{cc}2 L_{0} & 0 \\ 0 & 2 L_{1}-2\end{array}\right]$.

Another example is $H_{0}$ has even-even symmetry, $d_{0,0} \in \mathbb{Z}$ and $d_{0,1} \in \frac{1}{2} \mathbb{Z} \backslash \mathbb{Z} ; H_{1}$ has even-odd symmetry, $d_{1,0} \in \mathbb{Z}$ and $d_{1,1} \in$ $\frac{1}{2} \mathbb{Z} \backslash \mathbb{Z}$. This type of filter bank is compatible with symmetric extension with $f_{1}$ in (4) given by

$$
f_{1}\left[n_{1}\right]=\min \left\{\bmod \left(n_{1}, 2 L_{1}\right), 2 L_{1}-1-\bmod \left(n_{1}, 2 L_{1}\right)\right\} .
$$

With this symmetric extension, $x$ is quadrantally centrosymmetric about $\left[\begin{array}{cc}0 & -\frac{1}{2}\end{array}\right]^{T}$, and is $\boldsymbol{P}$-periodic with $\boldsymbol{P}=\left[\begin{array}{cc}2 L_{0}-2 & 0 \\ 0 & 2 L_{1}\end{array}\right]$.

\section{CONCLUSIONS}

In this paper, we have investigated how to preserve symmetry and periodicity under the convolution and downsampling operations of a quincunx filter bank. This led us to propose a new symmetric extension algorithm (and some minor variations thereof) which can be used to construct nonexpansive transforms associated with quincunx filter banks. This scheme is potentially useful in any application that processes finite-extent sequences using such filter banks.

\section{REFERENCES}

[1] M. J. T. Smith and S. L. Eddins, "Analysis/synthesis techniques for subband image coding," IEEE Trans. on Acoustics, Speech, and Signal Processing, vol. 38, no. 8, pp. 1446-1456, Aug. 1990.

[2] C. M. Brislawn, "Preservation of subband symmetry in multirate signal coding," IEEE Trans. on Signal Processing, vol. 43, no. 12, pp. 3046-3050, Dec. 1995.

[3] E. Viscito and J. P. Allebach, "The analysis and design of multidimensional FIR perfect reconstruction filter banks for arbitrary sampling lattices," IEEE Trans. on Circuits and Systems, vol. 38, no. 1, pp. 29-41, Jan. 1991.

[4] S. Coulombe and E. Dubois, "Linear phase and symmetries for multidimensional FIR filters over lattices," IEEE Trans. on Circuits and Systems-II: Analog and Digital Signal Processing, vol. 45, no. 4, pp. 473-481, Apr. 1998.

[5] D. E. Dudgeon and R. M. Mersereau, Multidimensional Digital Signal Processing, Prentice-Hall, Englewood Cliffs, NJ, USA, 1984.

[6] Y. Chen, M. D. Adams, and W.-S. Lu, "Symmetric extension for quincunx filter banks," in Proc. of IEEE Pacific Rim Conference on Communications, Computers and Signal Processing, Victoria, BC, Canada, Aug. 2005, to appear. 